\title{
SwissDRG Version 8.0: Nachvollziehbarkeit künftig fördern
}

\author{
Mirjam Bacha, Beatrix Meyer ${ }^{\mathrm{b}}$ \\ ${ }^{a}$ Dr. med., Expertin, $\mathrm{FMH}_{;}{ }^{\mathrm{b}}$ Leiterin Abteilung Stationäre Versorgung und Tarife, $\mathrm{FMH}$
}

\begin{abstract}
Das SwissDRG-System ist für die Anwenderinnen und Anwender zunehmend schwierig nachzuvollziehen. Dies einerseits deshalb, weil gleiche Themen immer wieder überarbeitet werden. Andererseits sind die DRGs zwar ökonomisch, jedoch häufig nicht medizinisch homogen. Verbesserungen konnte die SwissDRG AG bei der Abbildung von Hochdefizitfällen erzielen.
\end{abstract}

Für die Tarifversion 8.0 hat die SwissDRG AG erhebliche Umbauten vorgenommen. Ein Schwerpunkt ist dabei wie bereits in Version 7.0 die verbesserte Abbildung von hochaufwendigen defizitären Fällen. Dadurch reduzierte sich erfreulicherweise die Anzahl Fälle mit einem Defizit $>40000$ CHF. Das Defizitvolumen aller Spitäler sank im Vergleich zur SwissDRGVersion 6.0 um 28\%, beträgt aber trotzdem noch über 120 Mio. CHF. Im Fokus standen ausserdem erneut die Intensivmedizin sowie unter anderem die DRGs der Kinder- und Jugendmedizin.

Um die Kontinuität in der Tarifstruktur zu fördern, sollte die Verwendung mehrerer Datenjahre verstärkt geprüft werden.

Die Gesamtzahl der DRGs in Version 8.0 (1037 DRGs) unterscheidet sich gegenüber der Vorversion 7.0 (1041 DRGs) zwar nur geringfügig. Insgesamt wurden in Version 8.0 jedoch 13 Basis-DRGs gelöscht und 40 neue DRGs geschaffen. Die Anzahl der Zusatzentgelte zur Vergütung von teuren Medikamenten, Blutprodukten, Implantaten und Verfahren erhöhte sich auf insgesamt 108. Zudem wurden bereits bestehende Zusatzentgelte teilweise differenziert, z.B. durch die Ergänzung weiterer Dosisklassen.

\section{Thematische Umbauten innerhalb einer Tarifversion abschliessen}

Einige der bereits in SwissDRG-Version 7.0 bearbeiteten Themen hat die SwissDRG AG für die Version 8.0 nochmals revidiert. Sie baute mehrfach dieselben DRGs in zwei oder mehr aufeinanderfolgenden Tarifversionen um. In der Anwendung sind diese Anpassungen teilweise schwierig nachzuvollziehen. Dies gilt insbeson- dere, da das Fallpauschalensystem mit jeder Version komplexer wird. Diese Umstände erschweren den Spitälern zunehmend die Planung. Vorteilhaft wäre es, die Themen möglichst innerhalb der gleichen DRG-Version abschliessend zu bearbeiten. Die FMH empfiehlt, zunächst die Auswirkungen vorausgegangener Umbauten zu analysieren, bevor erneute Änderungen an denselben DRGs erfolgen. Die wiederholten Umbauten an den jeweils gleichen DRGs dürften allerdings auch mit der noch ungenügenden Datenqualität zusammenhängen. Hilfreich wäre es, wenn die Umbauten und deren Hintergründe in einem ausführlichen transparenten Bericht dokumentiert würden.

\section{Qualität und Quantität der Daten bleiben eine Herausforderung}

Die Güte des SwissDRG-Systems hängt massgeblich von der Datengrundlage ab. Deshalb unterstützt die SwissDRG AG die Spitäler, damit sich deren Kostenund Leistungsdaten kontinuierlich verbessern. Sie intensivierte den Austausch mit den Spitälern und bietet diesen die Möglichkeit, die Daten unter dem Jahr zu plausibilisieren. Ausserdem führte sie gezielte Workshops mit ausgewählten Kliniken durch. Trotz diesen Anstrengungen bleibt die Verbesserung der Datenqualität gemäss der SwissDRG AG eine Herausforderung. Abgesehen von der Datenqualität ist auch eine breite Datenbasis für die Systemstabilität wichtig. Wie die Abbildung zeigt, weisen jedoch mehrere DRGs für die aktuell angewendete SwissDRG-Version 7.0 nur eine geringe Kalkulationsbasis auf. Die SwissDRG AG berücksichtigt deshalb für fallzahlschwache DRGs jeweils zusätzlich die Daten vorangehender Jahre. ${ }^{1}$ In der SwissDRG-Version 8.0 fasste die SwissDRG AG für die Kalkulation von 30 fallzahlschwachen DRGs zwei oder 


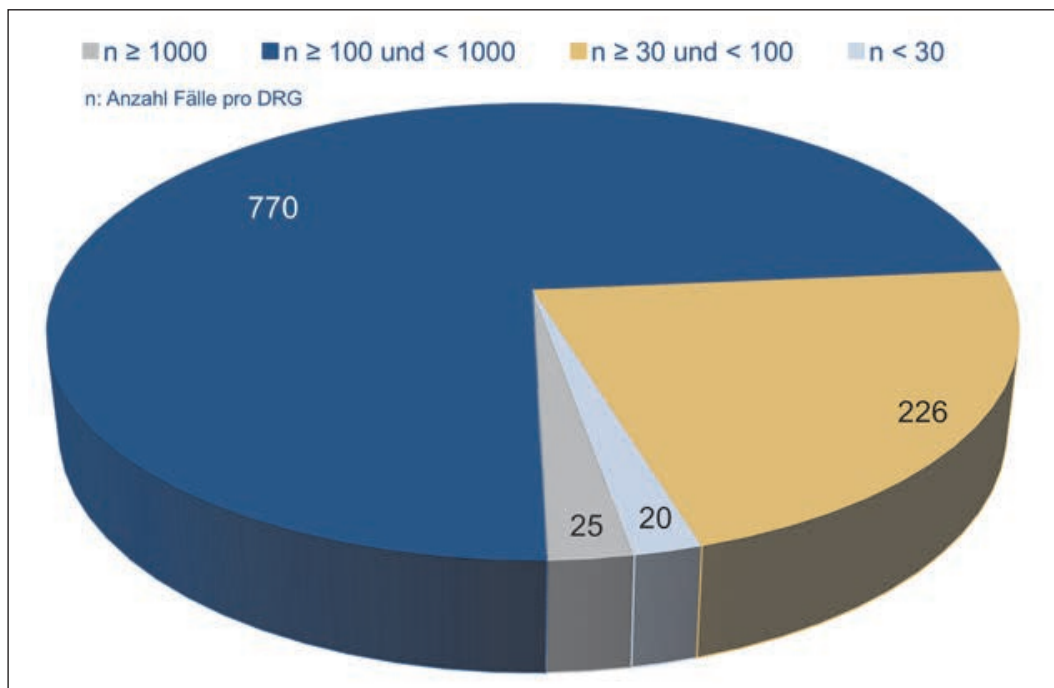

Beispiel: 20 DRGs wurden mit einer Datenbasis von weniger als 30 Fällen pro DRG kalkuliert.

Gesamtmenge der DRGs aufgeteilt nach Anzahl der Fälle als Kalkulationsbasis.

drei Datenjahre zusammen. Dieses Vorgehen sollte aus Sicht der FMH auch für fallzahlstärkere DRGs geprüft werden. Dadurch könnten die teilweise grossen Schwankungen der Kostengewichte zwischen den SwissDRG-Versionen reduziert werden. Die Kontinuität in der Tarifstruktur liesse sich fördern.

\section{Medizinische Homogenität verstärkt beachten}

Aufgrund der geringen Fallzahlen hat die SwissDRG AG medizinisch unterschiedliche Fälle teilweise in einer sich dies beispielsweise durch die Verwendung von mehreren Datenjahren vermeiden lässt. Aus Sicht der FMH ist es wichtig, nicht nur auf die ökonomische, sondern auch auf die medizinische Homogenität von DRGs zu achten. Das Zusammenfassen medizinisch vergleichbarer Leistungen in einer DRG ist eine wichtige Voraussetzung, damit das SwissDRG-System wickeln kann.

2 PCCL: patient-related complication or comorbidity level.

Korrespondenz: FMH Baslerstrasse 47 CH-4600 Olten Tel. 0313591111 Fax 0313591112 tarife.spital[at]fmh.ch tungen vorrangig in den organbezogenen Major Diagnostic Categories (MDCs) abgebildet werden. Den MDCs vorgeschaltet ist die sogenannte Prä-MDC. Diese umfasst besonders kostenintensive Fallgruppen (z.B. Transplantationen, Langzeitbeatmungen, Frührehabilitationen). Eine erste Umverteilung von Leistungen aus der Prä-MDC in die organbezogenen MDCs erfolgte bereits für die Version 7.0. In der Version 8.0 führte die SwissDRG zusammengefasst. Hier gilt es zu prüfen, ob nachvollziehbar wird und sich konsistent weiterent-

DRG AG diese Arbeiten in einigen Bereichen fort Dieses Vorgehen sollte weiterverfolgt werden, denn dadurch wird das SwissDRG-System übersichtlicher.

\section{Präzisere Abbildung der Komplexität im Einzelfall}

Um die Komplexität der medizinischen Krankheitsbilder darzustellen, arbeitet das DRG-System mit patientenbezogenen Schweregraden, den sogenannten PCCLStufen $^{2}$. Entsprechend der Einordnung als leichte, mässig schwere, schwere oder äusserst schwere Komplikation und/oder Komorbidität reicht der Wert von 0 bis 4. Bedauerlicherweise hat die SwissDRG AG den Antrag auf eine weitergehende Differenzierung durch Einführung der PCCL-Stufen 5 und 6 abgelehnt. Sie begründete dies mit technischer Nicht-Machbarkeit. Das deutsche DRG-System jedoch wendet diese Erweiterung bereits seit Jahren an. Die FMH bittet darum, die technischen Anforderungen für die Umsetzung erneut zu überprüfen - im Wissen um die technische und operative Komplexität des Anliegens.

\section{Fazit der FMH}

Mit den neuerlichen Umbauten verstärkt sich die Komplexität des SwissDRG-Systems weiter. Entsprechend wird die Tarifstruktur immer schwieriger nachvollziehbar. Dieser Problematik kann die SwissDRG AG begegnen, indem sie künftig die medizinische Homogenität von DRGs vermehrt beachtet. Zudem gilt es, themenbezogene Umbauten möglichst innerhalb einer

Es ist wichtig, nicht nur auf die ökonomische, sondern auch auf die medizinische Homogenität von DRGs zu achten.

SwissDRG-Version abzuschliessen. Ferner könnte das SwissDRG-System durch das Zusammenfassen von mehreren Datenjahren auch bei fallzahlstärkeren DRGs an Kontinuität gewinnen. Dies würde die Planung für die Spitäler deutlich erleichtern. Eine transparente Dokumentation wäre zudem für die Anwenderinnen und Anwender hilfreich.

\section{Bildnachweis}

Grafik erstellt von der FMH, Datenquelle: Datenspiegel der SwissDRG AG, SwissDRG-Version 7.0

Die detaillierte Einschätzung der FMH zur SwissDRG-Version 8.0 finden Sie unter: www.fmh.ch $\rightarrow$ Stationäre Tarife $\rightarrow$ Positionen $\rightarrow$ Stellungnahmen. 